\title{
Neural Substrates of Forward and Backward Associative Priming: A Functional MRI Study
}

\author{
Sarah Terrien ${ }^{1,2^{*}}$, Fabien Gierski ${ }^{1,2,3}$, Stéphanie Caillies ${ }^{1}$, Véronique Baltazart ${ }^{1}$, \\ Christophe Portefaix ${ }^{3}$, Laurent Pierot ${ }^{3}$, Chrystel Besche-Richard ${ }^{1,4}$ \\ ${ }^{1}$ Université de Reims Champagne-Ardenne, Laboratoire Cognition, Santé, Socialisation C2S EA 6291, Reims, France \\ ${ }^{2}$ Service de Psychiatrie des Adultes, Hôpital Robert Debré, CHU de Reims, Reims, France \\ ${ }^{3}$ Pôle d'Imagerie Médicale, Hôpital Maison-Blanche, CHU de Reims, Reims, France \\ ${ }^{4}$ Institut Universitaire de France, Paris, France \\ Email: "terriensarah@gmail.com
}

Received August $7^{\text {th }}, 2013$; revised September 11 $1^{\text {th }}, 2013$; accepted September $29^{\text {th }}, 2013$

Copyright (C) 2013 Sarah Terrien et al. This is an open access article distributed under the Creative Commons Attribution License, which permits unrestricted use, distribution, and reproduction in any medium, provided the original work is properly cited.

\begin{abstract}
Forward associative priming results of an association moves from the prime to the target whereas backward associative priming results of an association from the target to the prime (Koivisto, 1998). Little is known about this dissociation of process and the associated cerebral substrates. Fourteen healthy participants were included in this study. The task consisted in a lexical decision task using an fMRI-adapted semantic priming paradigm. Contrasts between forward related and forward unrelated conditions showed activation in the left temporal gyrus, left inferior prefrontal cortex, fusiform gyrus and occipital regions and cerebellum. Investigation of the different patterns of activation between forward and backward priming shows significant results: during the contrast between the forward priming effect and the backward priming effect, we observe a deactivation of BOLD response in temporal and frontal areas, which may reflect the post-lexical integration process. So, areas responsible for language and for decoding spelling seem not to be involved in the backward process. An adaptation of this research in event-related brain potentials is underway to better explore the temporality of post-lexical process.
\end{abstract}

Keywords: Semantic Memory; Forward and Backward Priming; fMRI; Left Superior Temporal Gyrus; Post-Lexical Integration

\section{Introduction}

Semantic priming is a well-described phenomenon in which a target word (e.g., flower) is recognized faster when it is preceded by a semantically related word (e.g., tree) than when it is preceded by an unrelated word (e.g., knife) (Meyer \& Schvaneveldt, 1971; Neely, 1991). Researchers have typically used the lexical decision task to observe the priming effect. In this task, a word (the prime) is presented visually for a fraction of a second, followed after a delay by a letter-string (the target). Participants have to judge whether or not the target is a word in their native language. The main semantic priming effects are known to involve three mechanisms: automatic spreading activation (ASA), expectancy generation (EG) and semantic matching (SM) (Neely, Keefe, \& Ross, 1989). The two first kinds of processes are considered to be pre-lexical processes and the third to be a post-lexical process. Several types of semantic priming have been identified in the literature, in particular forward associative priming and backward associative priming (Koriat, 1981). In forward associative priming, there is a diffusion of activation from the prime to the target, but not the other way around. In contrast, backward associative priming occurs as a result of a strong associative link moving from the target to the prime, but not the other way around (Koivisto,

\footnotetext{
*Corresponding author.
}

1998). Forward priming is presumably produced by pre-lexical processes, ASA at short stimulus onset asynchronies and EG at long ones (Franklin, Dien, Neely, Huber, \&Waterson, 2007). In contrast, it has been shown that backward priming requires a post-lexical process occurring when an association links the target to the prime but not vice versa, and may be due to SM and not to EG or ASA (Chwilla, Hagoort, \& Brown, 1998; Kahan, Neely, \& Forsythe, 1999).

Numerous studies have investigated the brain regions subserving semantic priming. As we would describe in more detail below, researchers first examined the overall mechanism of semantic priming compared to other cognitive processes. They then distinguished between regions involved in the different priming conditions (related vs. unrelated word pairs). Some authors have also attempted to identify the cerebral regions that support pre-lexical and post-lexical processes (Franklin et al., 2007; Kandhadai \& Federmeier, 2010; O'Hare, Dien, Waterson, \& Savage, 2008).

Early studies were carried out on patients with brain injuries and provided inconsistent results. Some studies showed a preservation of the semantic priming effect in patients with posterior left hemisphere lesions (Blumstein, Milberg, \& Shrier, 1982; Hagoort, 1997), while others found a semantic priming deficit in patients with similar brain damage (Hagoort, 1993; Henik, Dronkers, \& Knight, 1993; Milberg, Blumstein, Katz, 
Gershberg, \& Brown, 1995). This divergence led investigators to study this process with neuroimaging tools. Thus, several research using different neuroimaging techniques (event-related potentials (ERP) and positron emission tomography (PET)) were conducted. They mainly revealed an activation of the anterior cingulate cortex (ACC) and the left temporal anterior regions: the anterior fusiform gyrus and the hippocampal complex (Mummery, Shallice, \& Price, 1999; Nobre, Allison, \& McCarthy, 1994; Nobre \& McCarthy, 1995). These studies relied on a contrast between a control task (decision letter) and a lexical decision task involving semantic priming (Mummery et al., 1999).

Beyond the investigation of the neural substrates underlying semantic priming in general, in comparison with other cognitive tasks, authors have been also interested in the cerebral activations obtained during the related and unrelated conditions of the semantic priming task. In a functional magnetic resonance imaging (fMRI) study, Rossel, Bullmore, Williams, and David (2001) found activation of the ACC, the posterior cingulate, the right insula and the right temporal gyrus when they contrasted semantically related vs. unrelated conditions. According to these authors, the activation of temporal regions could be explained by the maintenance of ASA when the prime and the target are related, whereas the ACC's activation could be due to its role in the inhibition of incorrect responses. Moreover, several studies using PET scanning and fMRI have shown similar results regarding the reduction of activation in the left temporal cortex (Copland et al., 2003; Mummery et al., 1999; Rossel et al., 2001) during the related condition in comparison with the unrelated condition. Using ERPs, Matsumoto, Iidaka, Haneda, Okada and Sadato (2005) showed a pattern of decreased N400 for related conditions in semantic priming. Thanks to the source localization, these authors determined that the observed N400 was probably generated by the left superior temporal gyrus and the left superior frontal gyrus. This observation is very interesting because we know that temporal areas have been found to be related to semantic memory. As mentioned above, an fMRI study (Copland et al., 2003) revealed a decrease in cerebral activation in the left inferior prefrontal cortex (LIPC) during the related condition in comparison with the unrelated condition. The LIPC is known to be involved in selecting among competing representations in semantic memory and in semantic retrieval when the required information cannot be accessed through strong pre-existing cue-target associations (Wagner, Paré-Blagoev, Clark, \& Poldrack, 2001).

Few studies have investigated the distinction between the neural substrates involved in forward associative priming and those involved in backward associative priming, and the results are inconclusive. For instance, an experimental study using presentation of stimuli in visual half-field by Koivisto (1998) suggests that forward priming occurs in the left hemisphere whereas backward priming seems to occur in both hemispheres or predominantly in the right one. The results of an ERP study supported this finding: Franklin et al. (2007) suggested that the observed generated left temporal N400 reflected forward processes, whereas the observed generated right parietal gyrus N400 reflected backward associated processes. However, Kandhadai and Federmeier (2010) suggested that both hemispheres were able to strategically enhance the processing of backward words. They showed that the contributions of the right and left hemispheres depended on the nature of the task (active vs. passive task). To the best of our knowledge, the only fMRI study that has investigated forward and backward priming in healthy participants used the co-registered ERP/fMRI method (O'Hare et al., 2008). They showed bilateral activation of right sulcus, left frontal gyrus and cerebellum for forward priming, whereas backward priming occurred in right-hemisphere regions (median frontal gyrus, occipital area and visual cortex). However, as mentioned by the authors, this study has some methodological weaknesses. Indeed, for the asymmetrically related word pairs, authors used compound words (e.g., fruit-fly) and unidirectional related words (e.g., stork-baby). In their view, the use of compound words could create a bias because it was likely that when participants processed a backward association with compounded words (fly-fruit) they visually rearranged the prime and the target to form a compound stimulus (fruit-fly).

The aim of this research was to investigate the neural substrates subserving forward and backward semantic priming among healthy participants. We decided to use an event-related fMRI paradigm with mixed related and unrelated prime-target word pairs, manipulating the forward and backward association with asymmetrically related pairs, and not with compound words, to maximize the post-lexical integration process.

\section{Methods}

\section{Participants}

Fourteen healthy participants $(50 \%$ females; mean age $=22.5$ were recruited from the department of psychology of the University of Reims Champagne-Ardenne and the University of Reims Hospital. All participants were right-handed according to the Edinburgh Inventory (Oldfield, 1971); they were native speakers of French, had normal or corrected to normal vision, and had no history of reading disabilities. They also had no past or present history of neurological or psychiatric disorders or alcohol/drug abuse or dependence, and were free of medication. All participants conformed to standard health and safety regulations regarding the use of MRI. The experimental design was submitted to the local ethics committee, which approved the study. All participants gave their written informed consent prior to the study.

\section{Stimuli and Design}

Stimuli consisted of 200 pairs of words: 25 forward pairs of related words, 25 backward pairs of related words, 50 pairs of unrelated words, and 100 pseudo-words. To select the related word pairs, we first administered a free association task to a sample of 390 students at University of Reims. We chose for the backward condition only pairs of words which showed a difference of occurrence of greater than $53.06 \%$, and with a strong relation between word A and word B (between 55.1\% and $91.5 \%$ ) but a weak relation between word $\mathrm{B}$ and word $\mathrm{A}$ (between $0 \%$ and $14.3 \%$ ) (e.g., word $\mathrm{A}=$ sock and word $\mathrm{B}=$ shoe). For the forward condition, we picked related word pairs and pseudo-words from a previous study (Besche et al., 1997).

Two lists of stimuli were drawn up according to the following rules: (1) the pseudo-words were the same in both lists; (2) each word that was part of a related pair presented in one list was presented in the corresponding unrelated condition in the other list; (3) corresponding pairs in the two lists had the same target word; (4) the unrelated primes had the same number of letters, the same first letter and the same usage frequency in the language as the word in the corresponding list (Database: 
Lexique 3, www.lexique.org). For example, in list 1, a related word pair was chaussure-lacet (shoe-lace) and in list 2 the corresponding unrelated word pair was colonel-lacet (colonellace).

Stimuli were presented in a fixed random order. Each trial took place as follows: the fixation point was presented in the center of the screen for $300 \mathrm{~ms}$, followed by a prime word for $200 \mathrm{~ms}$, a mask for $50 \mathrm{~ms}$ and the target word for $3500 \mathrm{~ms}$. The next fixation point appeared automatically after an interstimulus interval of $100 \mathrm{~ms}$. The inter-trial interval was 3650 $\mathrm{ms}$ (see Figure 1). The fMRI recording was carried out over two runs (each lasting 6 minutes and 8 seconds), with a short break between them. Each run contained 100 trials.

All stimuli were presented on a non-magnetic screen viewed by participants via a mirror mounted on the head-coil. Prime words were presented in white characters on a black background whereas targets were presented in pink characters on a black background.

E-Prime software (Psychology Software Tools, Pittsburgh, PA) was used for the presentation of stimuli and the recording of reaction times. Responses were made on an MR-compatible response pad. The participants had to press the button with their right index finger if the target word (written in pink) was a real word and with their right middle finger if the target word was a pseudo-word. Only correct answers were considered.

\section{Image Acquisition and Procedure}

Images were acquired using a 3-Tesla whole-body MRI scanner (Achieva, Philips Medical Systems, Best, The Netherlands) with an 8-channel head coil. Head motions were minimized with a forehead strap and a comfortable padding around the participant's head. For each participant, a T1-weighted anatomical image oriented parallel to the Brain AC-PC Line was first acquired using a fast field echo sequence (T1-FFE, TR $=252.758 \mathrm{~ms} ; \mathrm{TE}=2.30 \mathrm{~ms}$; flip angle $=80^{\circ} ; 32$ axial slices; slice thickness $=4.50 \mathrm{~mm}$; no gap; FOV $=240 \times 144 \times 240$ $\mathrm{mm}$; matrix $=268 \times 214$; and acquisition voxel size $=0.43 \times$ $0.43 \times 4.5 \mathrm{~mm}^{3}$ ). Functional data were acquired from an ascending slice $2 \mathrm{D}-\mathrm{T} 2 *$-weighted EPI sequence sensitive to blood-oxygen-level-dependent (BOLD) contrast. This sequence was acquired in the same axial plane as the T1-weighted structural images and had the following parameters: $2 \mathrm{D}-\mathrm{T} 2 *$ FFE-EPI; TR $=2000 \mathrm{~ms}$; TE $=33 \mathrm{~ms}$; flip angle $=90^{\circ} ; 32$ axial slices; slice thickness $=4.50 \mathrm{~mm}$; no gap; matrix $=80 \times 80$; FOV $=240 \times 240 \mathrm{~mm}^{3}$; acquisition voxel size $=3 \times 3 \times 4.5$ $\mathrm{mm}^{3}$. The functional volumes were collected during two functional sessions of 184 volumes). Finally, a high resolution

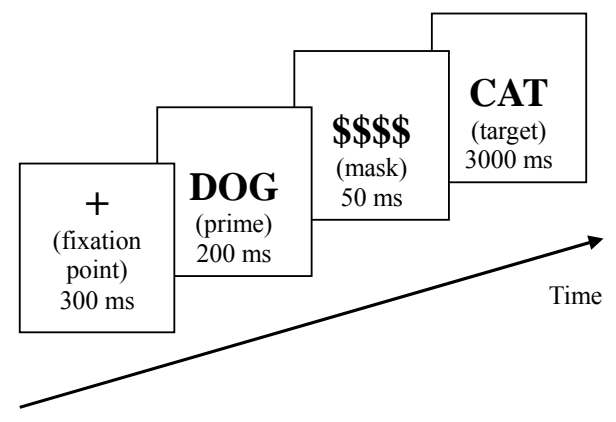

Figure 1.

Trial procedure.
T1-weighted anatomical image was acquired using a 3D turbo field echo sequence (3D-T1-TFE, TR $=8 \mathrm{~ms}$; TE $=3.73 \mathrm{~ms}$; flip angle $=8^{\circ} ; 160$ axial slices; slice thickness $=1 \mathrm{~mm}$; FOV $=$ $240 \times 240 \mathrm{~mm}$; matrix $=240 \times 240$; and acquisition voxel size $=1 \times 1 \times 1 \mathrm{~mm}^{3}$ ).

\section{fMRI Analyses}

The fMRI data were analyzed using Statistical Parametric Mapping (SPM8, Wellcome Department of Cognitive Neurology, Institute of Neurology, London, UK; www.fil.ion.ucl. ac.uk/spm/software/spm8). MRIcro software (www.micro.com) was used for image conversion. Five initial brain volumes of the functional run were discarded from the analyses to eliminate non-equilibrium effects of magnetization. Functional images were spatially realigned to the first volume, slice time corrected, and normalized to the standard space of the Montreal Neurological Institute (MNI) brain.

Images with excessive head movements $\left(>2 \mathrm{~mm}\right.$ or $\left.>2^{\circ}\right)$ were excluded from analyses. Spatial smoothing was done with an isotropic three-dimensional Gaussian filter with a full width at half maximum of $8 \mathrm{~mm}$. A high-pass filter was implemented using a cut-off period of 128 seconds to remove low-frequency drift from the time series.

For each participant, first-level contrast images were estimated for the following contrasts: forward related vs. forward unrelated, forward unrelated vs. forward related, backward related vs. backward unrelated and backward unrelated vs. backward related. The six movement parameters estimated during the realignment procedure were entered as regressors of non-interest into the model to control for the variance caused by micro-displacements of the head. Then, in order to examine our hypothesis, we used a second-level paired t-test with an extent threshold of 10 voxels and a statistical threshold of $\mathrm{P}<.001$, uncorrected, to explore differences between forward and backward priming activations. We made investigated the following contrasts: forward priming (forward related vs. forward unrelated) vs. backward priming (backward related vs. backward unrelated). The voxel coordinates of activations are reported in the MNI space in Table 1. Labeling was based on the AAL brain atlas (Tzourio-Mazoyer et al., 2002) using WFU PickAtlas (Maldjian, Laurienti, \& Burdette, 2004; Maldjian, Laurienti, Kraft, \& Burdette, 2003).

\section{Results}

\section{Behavioral Data}

Participants' mean reaction times are presented in Table 1. Two two-way ANOVAs with condition (unrelated/related) and type of relation (forward/backward) as factors were conducted with Statistica 7.1 on reaction times for correct answers for both participants (F1) and items (F2). The rate of correct answers was 98.96 (SD: 0.97). As expected, the analyses revealed a significant condition effect (related vs. unrelated; F1 $(1,13)=$

Table 1.

Mean reaction times (SD)(in milliseconds) for lexical decision.

\begin{tabular}{ccc}
\hline & Forward & Backward \\
\hline Related & $766(91.91)$ & $818(96.80)$ \\
Unrelated & $832(124.75)$ & $890(135.71)$ \\
\hline
\end{tabular}


$18.54 ; \mathrm{p}<.001 ; \mathrm{F} 2(1,98)=23.27 ; \mathrm{p}<.001)$, a significant effect of type of relation (backward vs. forward; F1 $(1,13)=$ $21.41 ; \mathrm{p}<.001 ; \mathrm{F} 2(1,13)=11.19 ; \mathrm{p}<.001)$, and no significant interaction $[\mathrm{F} 1(1,13)<1 ; \mathrm{F} 2(1,98)<1)$.

\section{fMRI Data}

The contrast between the forward unrelated condition and the forward related condition revealed significant activations (see Table 2). We observed bilateral activations of the inferior orbital frontal gyrus, the cingulum, and the medial and superior occipital gyrus. In the right hemisphere, we observed active tions of the fusiform gyrus, the cuneus, the cerebellum, the parahippocampalgyrus, the insula, the putamen, the medial occipitotemporalgyrus, and the lingual gyrus. In the left hemisphere we observed an activation of the superior temporal gyrus. The forward related $>$ forward unrelated contrast revealed no significant activation.

The contrast between the backward unrelated condition and the backward related condition revealed small activated clusters, with the largest having 26 voxels in the right cerebellum. The other two clusters were located in the left cerebellum (10 voxels) and the left medial occipital gyrus (14 voxels). The backward related $>$ backward unrelated contrast revealed no significant activation (see Table $\mathbf{3}$ ).

The contrast between the forward priming effect and backward priming effect revealed five significant clusters of activations. The largest cluster of activation was located in the left superior temporal gyrus (53 voxels), specifically in Wernicke's area (see Figure 2(a)). The other clusters were located in the right hemisphere in the temporal sub-gyrus, the medial frontal gyrus and precentralgyrus, the frontal sub-gyrus and the cingulate gyrus, the fronto-parietal gyrus, and the insula and putamen. The opposite subtraction revealed no significant difference.

We performed a plot estimation to dissociate the activation and deactivation under forward and backward priming. This analysis in each cluster revealed increased activation in the forward priming condition and deactivation in the backward priming condition (see Figure 2(b) and Table 4).

Table 2.

Results of fMRI forward priming effect.

\begin{tabular}{|c|c|c|c|c|c|}
\hline \multirow{2}{*}{ Anatomical regions } & \multicolumn{3}{|c|}{ Coordinates (MNI) } & \multirow[t]{2}{*}{ t-value } & \multirow[t]{2}{*}{ Number of voxels } \\
\hline & $\mathrm{X}$ & Y & $\mathrm{Z}$ & & \\
\hline Fusiform gyrus (R) & 42 & -50 & -6 & 5.89 & 63 \\
\hline Frontal sub-gyrus-Cingulate gyrus (R) & 22 & -24 & 32 & 5.66 & 58 \\
\hline Cingulum (R) & 20 & 14 & 36 & 4.96 & 53 \\
\hline Superior temporal gyrus (L) & -50 & -6 & -6 & 4.59 & 40 \\
\hline Superior and middle occipital gyrus-Cuneus (R) & 28 & -66 & 28 & 4.48 & 40 \\
\hline Inferior orbital frontal gyrus (R) & 38 & 34 & -8 & 4.37 & 27 \\
\hline Medial occipitotemporalgyrus-Cerebellum-Parahippocampalgyrus-Cuneus (R) & 26 & -52 & 4 & 4.34 & 209 \\
\hline Insula - Putamen ( R) & 34 & -8 & 2 & 4.33 & 20 \\
\hline Superior occipital gyrus (L) & -26 & -88 & 36 & 4.13 & 45 \\
\hline Cingulum (L) & -20 & -48 & 32 & 4.07 & 35 \\
\hline Inferior orbital frontal gyrus (L) & -24 & 32 & -12 & 3.99 & 12 \\
\hline Cerebellum (R) & 10 & -46 & -18 & 3.97 & 12 \\
\hline Medial occipitotemporalgyrus-Cuneus-Lingual gyrus (R) & 12 & -74 & 8 & 3.92 & 54 \\
\hline Medial occipital gyrus (L) & -40 & -82 & 2 & 3.92 & 19 \\
\hline Superior occipital gyrus (R) & 22 & -82 & 2 & 3.89 & 12 \\
\hline
\end{tabular}

Table 3.

Results of fMRI backward priming effect.

\begin{tabular}{cccccc}
\hline & & \multicolumn{2}{c}{ Coordinates (MNI) } & t-value & Number of voxels \\
\cline { 2 - 6 } Anatomical regions & $\mathrm{X}$ & $\mathrm{Y}$ & $\mathrm{Z}$ & & \\
\hline Inferior occipital gyrus - Middle temporal gyrus (R) & 48 & -80 & -2 & 4.41 & 14 \\
Cerebellum (R) & 34 & -54 & -26 & 4.18 & 26 \\
Cerebellum (L) & -30 & -54 & -26 & 3.61 & 10 \\
\hline
\end{tabular}




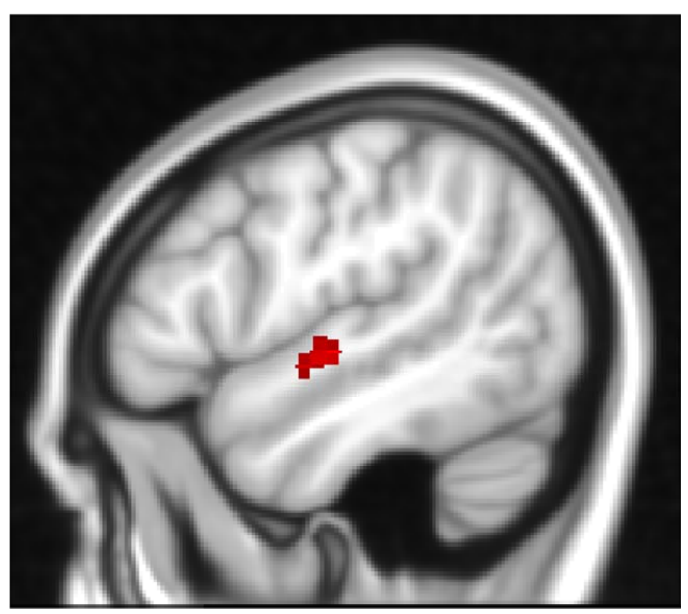

(a)

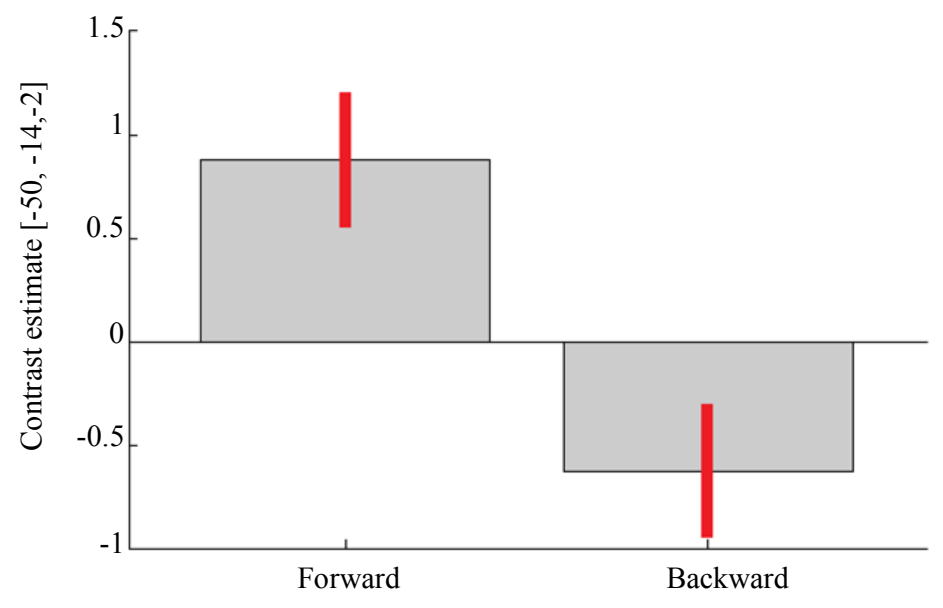

(b)

Figure 2.

Brain activation during forward and backward priming. (a) Activation to the effect of Forward $>$ Backwardconditions displayed on a $1 \mathrm{~mm}$ isotropic version of the MNI152 (Montreal Neurological Institute) standard brain (threshold: $\mathrm{p}<.001$ uncorrected). (b) Activation in the cluster is illustrated graphically with extracted estimate of BOLD signal change during priming.

Table 4.

Results of fMRI difference in priming effect.

\begin{tabular}{lccccc}
\hline \multirow{2}{*}{ Anatomical regions } & \multicolumn{2}{c}{ Coordinates (MNI) } & t-value & Number of voxels \\
\cline { 2 - 5 } & $\mathrm{X}$ & $\mathrm{Y}$ & $\mathrm{Z}$ & & 53 \\
\hline Superior temporal gyrus (L) & -50 & -14 & -2 & 5.39 & 23 \\
Temporal Sub-gyrus (R) & 42 & -50 & -4 & 5.34 & 47 \\
Medial frontal gyrus-Precentralgyrus (R) & 6 & -18 & 52 & 4.83 & 48 \\
Frontal sub-gyrus-Cingulate gyrus (R) & 22 & -24 & 34 & 4.77 & 24 \\
Fronto-parietal gyrus (R) & 24 & -32 & 50 & 4.28 & 22 \\
Putamen-Insula (R) & 34 & -8 & 2 & 4.15 & \\
\hline
\end{tabular}

\section{Discussion}

In this research, we studied forward semantic integration and backward semantic integration with an original semantic priming paradigm that had not previously been used in an fMRI study. A similar paradigm was used in an ERP study (Franklin et al., 2007) and in a co-registered ERP/fMRI study (O'Hare et al., 2008). The interesting feature of our study was that it used unilaterally linked words for the backward related pairs to maximize the post-lexical integration process in this condition. Behavioral analysis showed a significant priming effect in both forward and backward conditions, with no significant interaction. Franklin et al. (2007) did not mention any difference between backward and forward priming. These behavioral results suggest there is no difference in spreading activation between pre- and post-lexical processes. However, the functional results showed different patterns of activation in the forward and backward conditions.

\section{Forward Priming}

The activation contrast between the forward unrelated and forward related conditions highlighted several cerebral areas but we can consolidate some of them. Some of the brain regions activated were not predicted but were understandable given their role in certain cognitive functions. According to Pulvermüller (2012), different brain regions not necessarily directly involved in language interact in the treatment of the meaning of words in the human mind. First of all, we saw activation of the occipital regions: bilateral superior gyrus, medial occipital gyrus and cuneus. Activation of the superior gyrus and medial occipital gyrus bilaterally had been found in another semantic priming study (Rossel et al., 2003). It may be explained by the fact that during presentation of a target word that is unrelated to the prime, participants may need more time for lexical decision-making reflecting a more complex cognitive process than in the related condition. It is possible that this lexico-semantic processing requires more involvement of visual areas to recognize orthographic patterns. And the cuneus is a brain region of the striatal cortex, which projects visual information to the extrastriatal cortex and is implicated in pattern recognition and visual attention.

In our study, we found activation in two brain regions that had been identified as being mainly involved in semantic priming: the ACC and the LIPC, located in the medial orbital frontal gyrus (Copland et al., 2003; Matsumoto et al., 2005). Reduced 
activation of the right ACC in the related condition reveals a high level of perceptual abstraction during the presentation of unrelated words because these cerebral regions play a role in the representation of perceptual artifacts and living creatures (Gainotti, 2006). This reduction in activation is consistent with other studies (Copland et al., 2003; Matsumoto et al., 2005; Mummery et al., 1999; Rossel et al., 2001) and can be explained by the ACC's role in the inhibition of incorrect answers in decision-making (Rossel et al., 2001). Indeed, in the forward unrelated condition, the presentation of the target leads to incorrect EG, which must be inhibited by the participant. The activation in the LIPC during the unrelated condition reflects its involvement in semantic processing: its role is to select and retrieve the correct representation in semantic memory when the right information is not accessible through an existing association (Copland et al., 2003). If the prime and the target are related, it might appear that the subject does not need to select the correct answer or create a new association in memory. However, in the unrelated condition, the association does not exist, so subjects need to search for associations in semantic memory, which calls upon the LIPC.

The activation of the posterior cingulate cortex in the unrelated condition can be explained by its role in the visuospatial processing of stimuli, similarly to the implication of the occipital regions. The fusiform gyrus is normally known for its involvement in face recognition, but we also know that it is necessary for the recognition of complex shapes (Simon, Koutstaal, Pince, Wagner, \&Schacter, 2003). Reduced activation in this region during related pair processing shows again that word recognition is facilitated when the prime and the target are connected. Indeed, in the unrelated condition, participants must make an effort to understand and integrate the word presented, whereas in the related condition it is the process of semantic priming that allows recognition of the word, without going through a word decoding phase. This decrease of activation in Wernicke's area when identifying the target as a word in the related condition may be explained by the fact that in this condition the participant does not have to implement processing involving understanding or semantic memory because the semantic priming effect facilitates the answer. The activation of the lingual gyrus in the unrelated condition prompts a similar conclusion: subjects mobilize the cerebral zones responsible for word recognition.

Activation of the right cerebellum in the unrelated condition may be explained by the plausible implication of the posterolateral region of this cerebral structure in associative relations (Gebhart, Petersen, \& Thach, 2002). Moreover, this region seems to be involved in the generation of correct predictions concerning the relation between two stimuli in multiple processes (Bellebaum \& Daum, 2011; Timmann et al., 2010). A transcranial magnetic stimulation study showed that stimulation of the cerebellum improves lexical associative priming (Argyropoulos, 2011). So it is possible that this region is more solicited in a condition where no semantic relation exists between two stimuli and the participant fails to find a semantic matching association. As Sabb, Bilder, Chou, and Bookheimer (2007) observed in an fMRI study, the insula is activated during indirect priming, reflecting the role of this structure in working memory and attention. Another interesting study highlights this brain region's involvement in a search for semantic alternatives (Ketteler, Kastrau, Vohn, \& Huber, 2008), which may be illustrated here in the failed semantic matching between the prime and the target in the unrelated condition. The ventral occipitotemporal gyrus, and more specifically the extrastriatal cortex, is involved in visual word recognition and a lesion there causes repetition in the reading process (Behrmann, Nelson, \& Sekuler, 1998; Philipose et al., 2007; Starrfelt, Habekost, \& Leff, 2009); this might explain the activation of this region in our study. Sass, Krach, Sachs, and Kircher (2009) also found activation of the right putamen in this experimental condition. Daselaar et al. (2001) demonstrated the role of the medial temporal lobe in semantic retrieval thanks to activation of the right parahippocampal region, which might explain the activation of this region in the "unrelated" condition, in which participants had to recognize a word without help from semantic priming. Several clusters of activation identified in our study are brain areas that the Rossel et al. (2001) study identified as responsible for decision-making without lexical priming effects, such as the lingual gyrus, the fusiform gyrus, and Wernicke's area.

\section{Backward Priming}

The literature about the locus of activation of backward priming is neither abundant nor unanimous. An ERP study (Franklin et al., 2007) conducted with the divided visual field presentation (Koivisto et al., 1998) and an fMRI study (O'Hare et al., 2008) agreed that activation was localized in the right hemisphere during post-lexical processing. On the other hand, Kandhadai and Federmeier (2010) suggested that hemispheric lateralization was due not to the type of lexical processing (forward vs. backward) but to the nature of the task (passive vs. active). In our study, we found three clusters of activation, two of which were located in the right hemisphere. The activation of the right inferior occipital gyrus and the middle temporal gyrus in the same cluster reflects the activation of the associative visual cortex, which allows for pattern recognition. Activation in the right occipital area was also noted by Franklin et al. (2007). The involvement of these occipital and temporal regions might be explained by the process of orthographic word recognition, but this suggestion is speculative. Bilateral cerebellar activations are found in many functional imaging studies and lesion case studies report cerebellar involvement in language (Gordon, 1996; Leiner, Leiner, \& Dow, 1993; Marien, Engelborghs, \& De Deyn, 2001; Schmahmann \& Pandya, 1997). It is not clear whether this activation is specific to backward priming. That is why we choose to contrast forward priming activations and backward priming activations to see the real difference in activation between these two priming conditions.

\section{Forward Priming $>$ Backward Priming}

Five clusters of activations appeared when we contrasted forward priming and backward priming. These regions should represent areas responsible for pre-lexical priming. The activations were essentially located in temporal and frontal regions. The activation in the left superior temporal gyrus (Wernicke's area) shows that pre-lexical processing uses that classical language area. Other cerebral regions involved are known to subserve word recognition, such as the middle cingulate gyrus, which is responsible for the deletion of irrelevant information and decision-making, and the medial frontal and precentralgyri and particularly the posterior cingulate cortex, which performs evaluative functions. Right insula activation has been found in the locus of activation responsible for the priming process in 
Rossel et al.'s (2001) fMRI research, while Sass et al. (2009) find activation in the putamen. The activation in the right frontal sub-gyrus can be explained by its connection with the insula, which is also activated in this contrast. Moreover, this structure is the only part of the frontal lobe with a link to the insula (Catani et al., 2012), so this finding may reflect the spreading of activation between these two structures during the lexical decision task. It should be noted that the central sub-gyrus is a structure that straddles several lobes and can be considered as an extension of the somato-sensory primary cortex. So, the activation in the right temporal sub-gyrus is certainly linked to that in the frontal sub-gyrus and insula. Activation of the premotor cortex does not appear to be specifically related to pre-lexical semantic priming but most likely relates to the participant's motor response.

The most interesting result of this study is that all clusters in which we find activation for the forward $>$ backward priming contrast manifested activation in the forward condition and deactivation in the backward condition. This means that there is a real difference in the processes involved in pre- and postlexical semantic priming. Indeed, areas responsible for language and for decoding spelling (fusiform gyrus) seem to be not involved in the backward process. The reduction in BOLD response in the temporal and frontal regions when the related condition is compared to the unrelated condition reflects a decrease in the neuronal activity necessary to recognize words. Indeed, lexical decisions are easier and faster in the related condition because the presentation of the prime decreases the amount of activation required to recognize the related target word. This process involved is ASA (Stowe et al., 1999). Copland et al. (2003) speculated that this decrease in neuronal activity was the consequence of better post-lexical integration. In their view, the temporal resolution of fMRI cannot validate this hypothesis. However, in our study we manipulated the verbal material to make a difference between pre- and post-lexical processes, and the observations of Copland et al. seemed to be consistent with our results. The finding in our study of the deactivation of the BOLD response in the temporal and frontal areas in the backward condition is consistent with speculation by Copland et al. that post-lexical processing is necessary in the backward priming condition.

In summary, contrasts between experimental conditions show activation only during the subtraction of the related condition from the unrelated condition. No cerebral region is specific to semantic priming but most are involved in language processing (left temporal gyrus), decision-making (LIPC), pattern recognition (fusiform gyrus and occipital regions), and generation of correct predictions regarding the relation between two stimuli (cerebellum). Indeed, these cerebral areas are responsible for different cognitive processes that may be called upon during lexical decision-making when the prime and the target are not related (Copland et al., 2003; Mummery et al., 1999; Rossel et al., 2001). In this condition, the participant has to make an effort to understand and integrate the presented word, while in the related condition the presentation of the prime facilitates the recognition of the target word. Thus, the recognition process is easier and does not require a complex cognitive process. Another interesting finding of this research is the deactivation of the BOLD response in temporal and frontal areas, which may reflect post-lexical integration.

A limitation on our study is that prime-target pairs for the forward condition are not asymmetrical; consequently, we in- tend to carry out a new experiment with only asymmetrical pairs to make the backward and forward condition material more uniform. We plan to adapt this experiment to event-related brain potentials to better explore the temporality of backward and forward processes.

\section{REFERENCES}

Argyropoulos, G. (2011). Cerebellar theta-burst stimulation selectively enhances lexical associative priming. Cerebellum, 10, 540-550. http://dx.doi.org/10.1007/s12311-011-0269-y

Behrmann, M., Nelson, J. J., \& Sekuler, E. B. (1998). Visual complexity in letter-by-letter reading: "Pure" alexia is not pure. Neuropsychologia, 36, 1115-1132.

http://dx.doi.org/10.1016/S0028-3932(98)00005-0

Bellebaum, C., \& Daum, I. (2011). Mechanisms of cerebellar involvement in associative learning. Cortex, 47, 128-136. http://dx.doi.org/10.1016/j.cortex.2009.07.016

Besche, C., Passerieux, C., Segui, J., Sarfati, Y., Laurent, J. P., \& Hardy-Baylé, M. C. (1997). Syntactic and semantic processing in schizophrenic patients evaluated by lexical-decision tasks. Neuropsychology, 4, 498-505. http://dx.doi.org/10.1037/0894-4105.11.4.498

Blumstein, S. E., Milberg, W., \& Shrier, R. (1982). Semantic processing in aphasia: Evidence from an auditory lexical decision task. Brain and Language, 17, 301-315. http://dx.doi.org/10.1016/0093-934X(82)90023-2

Catani, M., Dell'Acqua, F., Vergani, F., Malik, F., Hodge, H., Roy, P., et al. (2012). Short frontal lobe connections of the human brain. Cortex, 48, 273-291. http://dx.doi.org/10.1016/j.cortex.2011.12.001

Chwilla, D. J., Hagoort, P., \& Brown, C. M. (1998). The mechanism underlying backward priming in a lexical decision task: Spreading activation versus semantic matching. The Quarterly Journal of Experimental Psychology A: Human Experimental Psychology, 51A, 531-560. http://dx.doi.org/10.1080/027249898391521

Copland, D. A., de Zubicaray, G. I., McMahon, K., Wilson, S. J., Eastburn, M., \& Chenery, H. J. (2003). Brain activity during automatic semantic priming revealed by event-related functional magnetic resonance imaging. NeuroImage, 20, 302-310. http://dx.doi.org/10.1016/S1053-8119(03)00279-9

Daselaar, S., Rombouts, S., Veltman, D., Raaijmakers, J., Lazeron, R., \& Jonker, C. (2001). Parahippocampal activation during successful recognition of words: A self-paced event-related fMRI study. NeuroImage, 13, 1113-1120. http://dx.doi.org/10.1006/nimg.2001.0758

Franklin, M. S., Dien, J., Neely, H. N., Huber, E., \& Waterson, L. D. (2007). Semantic priming modulates the N400, N300, and N400RP. Clinical Neurophysiology, 118, 1053-1068. http://dx.doi.org/10.1016/j.clinph.2007.01.012

Gainotti, G. (2006). Anatomical functional and cognitive determinants of semantic memory disorders. Neuroscience and Biobehavioral Reviews, 30, 577-594.

http://dx.doi.org/10.1016/j.neubiorev.2005.11.001

Gebhart, A. L., Petersen, S. E., \& Thach, W. T. (2002). Role of the posterolateral cerebellum in language. In S. M. Highstein, \& W. Thach (Eds.), The cerebellum: Recent developments in cerebellar research (pp. 318-333). New York: New York Academy of Sciences.

Gordon, N. (1996). Speech, language, and the cerebellum. European Journal of Disorders of Communication, 31, 359-367. http://dx.doi.org/10.3109/13682829609031327

Hagoort, P. (1993). Impairments of lexical-semantic processing in aphasia: Evidence from the processing of lexical ambiguities. Brain and Language, 45, 189-232. http://dx.doi.org/10.1006/brln.1993.1043

Hagoort, P. (1997). Semantic priming in Broca's aphasics at a short SOA: No support for an automatic access deficit. Brain and Language, 56, 287-300. http://dx.doi.org/10.1006/brln.1997.1849

Henik, A., Dronkers, N. F., \& Knight, R. T. (1993). Differential effects of semantic and identity priming in patients with left and right hemisphere lesions. Journal of Cognition and Neuroscience, 5, 45-55. http://dx.doi.org/10.1162/jocn.1993.5.1.45 
Kahan, T. A., Neely, J. H., \& Forsythe, W. J. (1999). Dissociated backward priming effects in lexical decision and pronunciation tasks. Psychonomic Bulletin and Review, 6, 105-110.

http://dx.doi.org/10.3758/BF03210816

Kandhadai, P., \& Federmeier, K. D. (2010). Automatic and controlled aspects of lexical associative processing in the two cerebral hemispheres. Psychophysiology, 47, 774-785. http://dx.doi.org/10.1111/j.1469-8986.2009.00969.x

Ketteler, D., Kastrau, F., Vohn, R., \& Huber, W. (2008). The subcortical role of language processing. High level linguistic features such as ambiguity-resolution and the human brain: An fMRI study. NeuroImage, 39, 2002-2009.

http://dx.doi.org/10.1016/j.neuroimage.2007.10.023

Koivisto, M. (1998). Backward priming and postlexical processing in the right hemisphere. Laterality, 3, 21-40.

http://dx.doi.org/10.1080/135765098397386

Koriat, A. (1981). Semantic facilitation in lexical decision as a function of prime-target association. Memory and Cognition, 9, 587-598. http://dx.doi.org/10.3758/BF03202353

Leiner, H. C., Leiner, A. L., \& Dow, R. S. (1993). Cognitive and language functions of the human cerebellum. Trends in Neurosciences, 16, 444-447. http://dx.doi.org/10.1016/0166-2236(93)90072-T

Maldjian, J. A., Laurienti, P. J., \& Burdette, J. H. (2004). Precentralgyrus discrepancy in electronic versions of the Talairach atlas. NeuroImage, 21, 450-455.

http://dx.doi.org/10.1016/j.neuroimage.2003.09.032

Maldjian, J. A., Laurienti, P. J., Kraft, R. A., \& Burdette, J. H. (2003). An automated method for neuroanatomic and cytoarchitectonic atlasbased interrogation of fMRI data sets. NeuroImage, 19, 1233-1239. http://dx.doi.org/10.1016/S1053-8119(03)00169-1

Marien, P., Engelborghs, S., \& De Deyn, P. (2001). Cerebellar neuroncognition: A new avenue. ActaNeurologicaBelgica, 101, 96-109.

Matsumoto, A., Iidaka, T., Haneda, K., Okada, T., \& Sadato, N. (2005). Linking semantic priming effect in functional MRI and event-related potentials. NeuroImage, 24, 624-634. http://dx.doi.org/10.1016/j.neuroimage.2004.09.008

Meyer, D. E., \& Schvaneveldt, R. W. (1971). Facilitation in recognizing pairs of words: Evidence of a dependence between retrieval operations. Journal of Experimental Psychology, 90, 227-234. http://dx.doi.org/10.1037/h0031564

Milberg, W., Blumstein, S. E., Katz, D., Gershberg, F., \& Brown, T. (1995). Semantic facilitation in aphasia: Effects of time and expectancy. Journal of Cognition and Neuroscience, 7, 33-50. http://dx.doi.org/10.1162/jocn.1995.7.1.33

Mummery, C. J., Shallice, T., \& Price, C. J. (1999). Dual-process model in semantic priming: A functional imaging perspective. NeuroImage, 9, 516-525. http://dx.doi.org/10.1093/cercor/bhp055

Neely, J. H. (1991). Semantic priming effects in visual word recognition: A selective review of current findings and theories. In D. Besner, \& G. W. Humphreys (Eds.), Basic processes in reading: Visual word recognition (pp. 264-336). Hillsdale, NJ: Lawrence Erlbaum Associates, Inc.

Neely, J. H., Keefe, D. E., \& Ross, K. L. (1989). Semantic priming in the lexical decision task: Roles of prospective prime-generated expectancies and retrospective semantic matching. Journal of Experimental Psychology: Learning, Memory, and Cognition, 15, 10031019. http://dx.doi.org/10.1037/0278-7393.15.6.1003

Nobre, A. C., Allison, T., \& McCarthy, G. J. (1994).Word recognition in the human inferior temporal lobe. Nature, 372, 260-273. http://dx.doi.org/10.1038/372260a0

Nobre, A. C., \& McCarthy, G. J. (1995). Language-related field potentials in the anterior- medial temporal lobe: II. Effects of word type and semantic priming. Journal of Neuroscience, 15, 1090-2008.

O'Hare, A. J., Dien, J., Waterson, L. D., \& Savage, C. R. (2008). Activation of the posterior cingulate by semantic priming: A co-Registered ERP/fMRI study. Brain Research, 1189, 97-114. http://dx.doi.org/10.1016/j.brainres.2007.10.095

Oldfield, R. C. (1971). The assessment and analysis of handedness: The Edinburgh inventory. Neuropsychologia, 9, 97-114. http://dx.doi.org/10.1016/0028-3932(71)90067-4

Philipose, L. E., Gottesman, R. F., Newhart, M., Kleinman, J. T., Herskovits, E. H., Pawlak, M. A., et al. (2007). Neural regions essential for reading and spelling of words and pseudowords. Annals of Neurology, 62, 481-492. http://dx.doi.org/10.1002/ana.21182

Pulvermüller, F. (2012). Meaning and the brain: The neurosemantics of referential, interactive, and combinatorial knowledge. Journal of Neurolinguistics, 25, 423-459. http://dx.doi.org/10.1016/j.jneuroling.2011.03.004

Rossel, S. L., Bullmore, E. T., Williams, S. C. R., \& David, A. S. (2001). Brain activation during automatic and controlled processing of semantic relations: A priming experiment using lexical-decision. Neuropsychologia, 39, 1167-1176. http://dx.doi.org/10.1016/S0028-3932(01)00049-5

Sabb, F. W., Bilder, R. M., Chou, M., \& Bookheimer, S. Y. (2007). Working memory effects on semantic processing: Priming differences in pars orbitalis. NeuroImage, 37, 311-322. http://dx.doi.org/10.1016/j.neuroimage.2007.04.050

Sass, K., Krach, S., Sachs, O., \& Kircher, T. (2009). Lion-tiger-stripes: Neural correlates of indirect semantic priming across processing modalities. NeuroImage, 45, 224-236. http://dx.doi.org/10.1016/j.neuroimage.2008.10.014

Schmahmann, J. D., \& Pandya, D. N. (1997). The cerebrocerebellar system. International Review of Neurobiology, 41, 31-60. http://dx.doi.org/10.1016/S0074-7742(08)60346-3

Simon, J. S., Koutstaal, W., Pince, S., Wagner, A. D., \& Schacter, D. L. (2003). Neural mechanisms of visual object priming: Evidence for perceptual and semantic distinctions in fusiform cortex. NeuroImage, 19, 613-626. http://dx.doi.org/10.1016/S1053-8119(03)00096-X

Starrfelt, R., Habekost, T., \& Leff, A. (2009). Too little, too late: Reduced visual span and speed characterize pure alexia. Cerebral Cortex, 19, 2880-2890. http://dx.doi.org/10.1093/cercor/bhp059

Stowe, L. A., Paans, A. M., Wijers, A. A., Zwarts, F., Mulder, G., \& Vaalburg, W. (1999). Sentence comprehension and word repetition: A positron emission tomography investigation. Psychophysiology, 36, 786-801. http://dx.doi.org/10.1111/1469-8986.3660786

Timmann, D., Drepper, J., Frings, M., Maschke, M., Richter, S., Gerwig, M., et al. (2010). The human cerebellum contributes to motor, emotional and cognitive associative learning: A review. Cortex, 46, 845-857. http://dx.doi.org/10.1016/j.cortex.2009.06.009

Tzourio-Mazoyer, N. N., Landeau, B. B., Papathanassiou, D. D., Crivello, F. F., Etard, O. O., Delcroix, N. N., et al. (2002). Automated anatomical labeling of activations in SPM using a macroscopic anatomical parcellation of the MNI MRI single-subject brain. NeuroImage, 15, 273. http://dx.doi.org/10.1006/nimg.2001.0978

Wagner, A. D., Paré-Blagoev, E. J., Clark, J., \& Poldrack, R. A. (2001). Recovering meaning: Left prefrontal cortex guides controlled semantic retrieval. Neuron, 2, 329-338.

http://dx.doi.org/10.1016/S0896-6273(01)00359-2 